\title{
MINIMAL CLONES WITH MANY MAJORITY OPERATIONS
}

\author{
MIKE BEHRISCH AND TAMÁS WALDHAUSER \\ Dedicated to Béla Csákány on his eightieth birthday
}

\begin{abstract}
We present two minimal clones containing 26 and 78 majority operations respectively, more than any other previously known example.
\end{abstract}

\section{INTRODUCTION}

A clone is a family of finitary operations defined on a set $A$ that is closed under composition of functions and contains all projections (which will also be called trivial functions). Given a set $F$ of operations on $A$, the functions obtained from elements of $F$ and from projections by means of compositions form the smallest clone containing $F$. This is the clone generated by $F$, and we denote this clone by $[F]$. This clone is nothing else but the clone of term functions of the algebra $(A ; F)$.

The set of all clones on a given base set $A$ is a complete lattice; the largest element of this lattice is the clone of all operations on $A$, and the smallest element is the clone containing projections only. The latter is called the trivial clone, denoted by $\mathcal{I}$. A minimal clone is an atom in the clone lattice, i.e., a nontrivial clone, whose only proper subclone is $\mathcal{I}$. As opposed to the case of maximal clones (coatoms of the clone lattice), the description of minimal clones is still an open problem, although there are numerous partial results. Here we review only those facts about minimal clones that we need in the sequel, for an overview of minimal clones we refer the reader to the survey papers 3 and [9] for general reference on clones see [6, 8, 10.

It follows from the definition that every minimal clone is generated by any one of its nontrivial members, and a nontrivial function $f$ generates a minimal clone iff

$$
f \in[h] \text { holds for all } h \in[f] \backslash \mathcal{I} .
$$

We will consider clones generated by a majority operation, i.e., by a ternary operation $f$ satisfying

$$
f(a, a, b)=f(a, b, a)=f(b, a, a)=a \text { for all } a, b \in A .
$$

As it was shown in [2, in this case all ternary functions in $[f]$ are majority operations, except for the three projections.

For any clone $\mathcal{C}$, let $\mathcal{C}^{(3)}$ denote the set of ternary operations belonging to $\mathcal{C}$. The composition of functions yields a quaternary operation on $\mathcal{C}^{(3)}$ as we have one outer function and three inner functions in a composition. Furthermore, we may regard the three ternary projections in $\mathcal{C}^{(3)}$ as nullary operations. With these operations $\mathcal{C}^{(3)}$ becomes an algebra of type $(4,0,0,0)$, a so-called unitary Menger algebra of rank 3 (cf. [7). Among the many pleasant properties of majority operations, there is one that is especially useful in the investigation of minimal clones: if $f$ is a majority operation, then the minimality of the clone $\mathcal{C}=[f]$ is determined by its

2010 Mathematics Subject Classification. Primary: 08A40.

Key words and phrases. Clone, minimal clone, majority operation. 


\begin{tabular}{|c|c|ccc|cccccccc|}
\hline & $m_{1}$ & $m_{2}$ & & & $m_{3}$ & & & & & \\
\hline$(1,2,3)$ & 1 & 1 & 2 & 3 & 3 & 3 & 1 & 3 & 1 & 1 & 3 & 1 \\
$(2,3,1)$ & 1 & 2 & 3 & 1 & 3 & 1 & 3 & 3 & 1 & 3 & 1 & 1 \\
$(3,1,2)$ & 1 & 3 & 1 & 2 & 3 & 3 & 3 & 1 & 1 & 1 & 1 & 3 \\
\hline$(2,1,3)$ & 1 & 2 & 1 & 3 & 1 & 3 & 1 & 1 & 3 & 1 & 3 & 3 \\
$(1,3,2)$ & 1 & 1 & 3 & 2 & 1 & 1 & 1 & 3 & 3 & 3 & 3 & 1 \\
$(3,2,1)$ & 1 & 3 & 2 & 1 & 1 & 1 & 3 & 1 & 3 & 3 & 1 & 3 \\
\hline
\end{tabular}

TABLE 1. Majority operations generating a minimal clone on the three-element set $\{1,2,3\}$

ternary part, i.e., it suffices to check the minimality criterion (1) only for ternary functions (see $[2,12]$ ). Formally, if $f$ is a majority operation, then $[f]$ is a minimal clone iff

$$
f \in[h] \text { holds for all } h \in[f]^{(3)} \backslash \mathcal{I} .
$$

If the base set is finite, this means that there are only finitely many functions $h$ to be tested, hence, at least in principle, it can be done by computer.

There are very few examples of minimal clones generated by a majority operation (while there is an abundance of examples of other types of minimal clones). Two general examples are: the median function $(x \wedge y) \vee(y \wedge z) \vee(z \wedge x)$ on any lattice (see, e.g., 8]), and the dual discriminator function on any set (see [4, [5]). All other examples came from systematic investigations of minimal clones on small sets. B. Csákány determined all minimal clones on any three-element set in [1], and among the clones he found there are up to isomorphism three that are generated by a majority operation. These clones contain 1,3 and 8 majority operations, respectively; see Table 1. (Here, and in the other tables we omit those triples where the majority rule determines the values of the functions.)

Suppose that $f$ is a majority operation on $A$ generating a minimal clone. If $f$ is conservative, i.e., it preserves every subset of $A$, then the restriction of $f$ to any three-element subset has to be isomorphi ${ }^{1}$ to one of the 12 functions in Table 1 . and $f$ is uniquely determined by these restrictions. However, the converse is not true: given a conservative majority operation $f$ whose restriction to every threeelement subset is isomorphic to one of these 12 functions, it is not guaranteed that $[f]$ is a minimal clone. The appropriate necessary and sufficient condition for the minimality was given by B. Csákány in 2. It turns out that a minimal clone generated by a conservative majority operation contains either 1, 3, 8 or 24 majority operations. If both $m_{2}$ and $m_{3}$ appear among the restrictions of $f$, then all possible pairs of majority operations from $\left[m_{2}\right] \times\left[m_{3}\right]$ appear as restrictions of compositions of $f$; this yields 24 majority functions.

The investigation of minimal clones on the four-element set carried out in [11] did not give any new examples: if $f$ is a majority operation on a four-element set generating a minimal clone, then $f$ is either conservative, or it is isomorphic to one of the 12 functions shown in Table 2. (The middle two rows mean that the value of the functions on $(a, b, c)$ is 4 whenever $\{a, b, c\}=\{1,2,4\}$ or $\{a, b, c\}=\{1,3,4\}$.)

\footnotetext{
${ }^{1}$ By a slight abuse of terminology, we say that operations $f$ and $g$ defined on sets $A$ and $B$, respectively, are isomorphic, if the algebras $(A ; f)$ and $(B ; g)$ are isomorphic.
} 


\begin{tabular}{|c|c|ccc|cccccccc|}
\hline & $M_{1}$ & $M_{2}$ & & & $M_{3}$ & & & & & & \\
\hline$(1,2,3)$ & 4 & 4 & 2 & 3 & 3 & 3 & 4 & 3 & 4 & 4 & 3 & 4 \\
$(2,3,1)$ & 4 & 2 & 3 & 4 & 3 & 4 & 3 & 3 & 4 & 3 & 4 & 4 \\
$(3,1,2)$ & 4 & 3 & 4 & 2 & 3 & 3 & 3 & 4 & 4 & 4 & 4 & 3 \\
\hline$(2,1,3)$ & 4 & 2 & 4 & 3 & 4 & 3 & 4 & 4 & 3 & 4 & 3 & 3 \\
$(1,3,2)$ & 4 & 4 & 3 & 2 & 4 & 4 & 4 & 3 & 3 & 3 & 3 & 4 \\
$(3,2,1)$ & 4 & 3 & 2 & 4 & 4 & 4 & 3 & 4 & 3 & 3 & 4 & 3 \\
\hline$\{1,2,4\}$ & 4 & 4 & 4 & 4 & 4 & 4 & 4 & 4 & 4 & 4 & 4 & 4 \\
\hline$\{1,3,4\}$ & 4 & 4 & 4 & 4 & 4 & 4 & 4 & 4 & 4 & 4 & 4 & 4 \\
\hline$(4,2,3)$ & 4 & 4 & 2 & 3 & 3 & 3 & 4 & 3 & 4 & 4 & 3 & 4 \\
$(2,3,4)$ & 4 & 2 & 3 & 4 & 3 & 4 & 3 & 3 & 4 & 3 & 4 & 4 \\
$(3,4,2)$ & 4 & 3 & 4 & 2 & 3 & 3 & 3 & 4 & 4 & 4 & 4 & 3 \\
\hline$(2,4,3)$ & 4 & 2 & 4 & 3 & 4 & 3 & 4 & 4 & 3 & 4 & 3 & 3 \\
$(4,3,2)$ & 4 & 4 & 3 & 2 & 4 & 4 & 4 & 3 & 3 & 3 & 3 & 4 \\
$(3,2,4)$ & 4 & 3 & 2 & 4 & 4 & 4 & 3 & 4 & 3 & 3 & 4 & 3 \\
\hline
\end{tabular}

TABLE 2. Nonconservative majority operations generating a minimal clone on the four-element set $\{1,2,3,4\}$

Restricting these functions to $\{2,3,4\}$ we get (isomorphic copies of) the 12 functions of Table 1 and, in fact, this restriction is a clone isomorphism.

In all of the above examples, the clone contains $1,3,8$ or 24 majority operations, and actually the ternary part of the clone, as a Menger algebra, is determined up to isomorphism by its size (see [12] for details). This gives rise to the question whether this is always the case. Some modest steps have been taken in [12] to give an affirmative answer to this question. However, it turns out that the answer is negative: we will prove the following theorem in Section 2

Theorem 1. There exists a minimal clone with 26 majority operations.

The other main result of this paper is that the same "trick" that makes it possible to construct a minimal clone with 24 majority operations using $m_{2}$ and $m_{3}$ works with any majority operation $f$ in place of $m_{3}$, provided that $f$ is cyclically symmetric, i.e., $f$ satisfies the identity $f\left(x_{1}, x_{2}, x_{3}\right) \approx f\left(x_{2}, x_{3}, x_{1}\right)$.

Theorem 2. If there is a minimal clone with $n$ majority operations one of which is cyclically symmetric, then there is a minimal clone with $3 n$ majority operations.

Since the clone containing 26 majority operations that we present in Section 2 is generated by a cyclically symmetric majority operation, the above theorem implies that there is a minimal clone with 78 majority operations. In sum, what we know about the number of majority operations in a minimal clone is that it can be 1 , $3,8,24,26$ or 78 , but it cannot be 2 or 4 (cf. [12]). We do not know if there are infinitely many such numbers, and we do not even know whether every minimal clone contains only finitely many majority operations.

\section{Proof of Theorem 1}

Theorem 1 is a result of a computer search: we checked for each non-conservative cyclically symmetric majority operation $f$ on a five-element set whether $[f]$ is a minimal clone or not. We considered only cyclically symmetric functions, because 


\begin{tabular}{|c|c|c|c|c|c|c|c|c|c|c|}
\hline & $f_{1}$ & $f_{2}$ & $g_{1}^{u, v}$ & $g_{2}^{u, v}$ & $g_{3}^{u, v}$ & \multicolumn{3}{|c|}{$g_{4}^{u, v} g_{5}^{u, v} g_{6}^{u, v}$} \\
\hline & & $\{0,1, \overline{1}\}$ & 1 & 1 & 1 & 1 & 1 & 1 & 1 & 1 \\
\hline & & $\{2,1, \overline{1}\}$ & 1 & 1 & 1 & 1 & 1 & 1 & 1 & 1 \\
\hline & & $\{0,2, \overline{2}\}$ & 2 & 2 & 2 & 2 & 2 & 2 & 2 & 2 \\
\hline & & $\{1,2, \overline{2}\}$ & 2 & 2 & 2 & 2 & 2 & 2 & 2 & 2 \\
\hline & & $\{\overline{1}, 2, \overline{2}\}$ & 2 & 2 & 2 & 2 & 2 & 2 & 2 & 2 \\
\hline$(0, \overline{1}, 2)$ & $(0,1, \overline{2})$ & $(0,1,2)$ & 1 & 2 & 1 & 2 & 2 & 2 & 1 & 1 \\
\hline$(\overline{1}, 2,0)$ & $(1, \overline{2}, 0)$ & $(1,2,0)$ & 1 & 2 & 2 & 1 & 2 & 1 & 2 & 1 \\
\hline$(2,0, \overline{1})$ & $(\overline{2}, 0,1)$ & $(2,0,1)$ & 1 & 2 & 2 & 2 & 1 & 1 & 1 & 2 \\
\hline$(2, \overline{1}, 0)$ & $(\overline{2}, 1,0)$ & $(2,1,0)$ & 2 & 1 & 1 & 2 & 1 & 2 & 1 & 2 \\
\hline$(\overline{1}, 0,2)$ & $(1,0, \overline{2})$ & $(1,0,2)$ & 2 & 1 & 1 & 1 & 2 & 2 & 2 & 1 \\
\hline$(0,2, \overline{1})$ & $(0, \overline{2}, 1)$ & $(0,2,1)$ & 2 & 1 & 2 & 1 & 1 & 1 & 2 & 2 \\
\hline & & $(0, \overline{1}, \overline{2})$ & 1 & 2 & $\mathbf{u}$ & 2 & 2 & 2 & $\mathbf{v}$ & 1 \\
\hline & & $(\overline{1}, \overline{2}, 0)$ & 1 & 2 & 2 & $\mathbf{u}$ & 2 & 1 & 2 & $\mathbf{v}$ \\
\hline & & $(\overline{2}, 0, \overline{1})$ & 1 & 2 & 2 & 2 & $\mathbf{u}$ & $\mathbf{v}$ & 1 & 2 \\
\hline & & $(\overline{2}, \overline{1}, 0)$ & 2 & 1 & $\mathbf{v}$ & 2 & 1 & 2 & $\mathbf{u}$ & 2 \\
\hline & & $(\overline{1}, 0, \overline{2})$ & 2 & 1 & 1 & $\mathbf{v}$ & 2 & 2 & 2 & $\mathbf{u}$ \\
\hline & & $(0, \overline{2}, \overline{1})$ & 2 & 1 & 2 & 1 & $\mathbf{v}$ & $\mathbf{u}$ & 2 & 2 \\
\hline & & $(\overline{2}, 1, \overline{1})$ & 1 & $\overline{1}$ & 1 & $\overline{1}$ & $\overline{1}$ & $\overline{1}$ & 1 & 1 \\
\hline & & $(1, \overline{1}, \overline{2})$ & 1 & $\overline{1}$ & $\overline{1}$ & 1 & $\overline{1}$ & 1 & $\overline{1}$ & 1 \\
\hline & & $(\overline{1}, \overline{2}, 1)$ & 1 & $\overline{1}$ & $\overline{1}$ & $\overline{1}$ & 1 & 1 & 1 & $\overline{1}$ \\
\hline & & $(\overline{1}, 1, \overline{2})$ & $\overline{1}$ & 1 & 1 & $\overline{1}$ & 1 & $\overline{1}$ & 1 & $\overline{1}$ \\
\hline & & $(1, \overline{2}, \overline{1})$ & $\overline{1}$ & 1 & 1 & 1 & $\overline{1}$ & $\overline{1}$ & $\overline{1}$ & 1 \\
\hline & & $(\overline{2}, \overline{1}, 1)$ & $\overline{1}$ & 1 & $\overline{1}$ & 1 & 1 & 1 & $\overline{1}$ & $\overline{1}$ \\
\hline
\end{tabular}

TABLE 3. A minimal clone with 26 majority operations

the number of all majority operations is so huge, that the problem seems to be inaccessible. Even for the cyclically symmetric case, the task took several weeks on several computers. The outcome is that except for one function (up to isomorphism and up to permutation of variables) the minimal clones contain 1 or 8 majority operations, and the structure of the ternary part is the same as that of $\left[m_{1}\right]$ or $\left[m_{3}\right]$ (see Table 1). The exceptional function is the function $f_{1}$ in Table 3 it generates a minimal clone with 26 majority operations. This was first proven by computer, but it is possible to verify it by human reasoning as well (to be presented in this section). However, we do not have a "human" proof for the fact that this is the only cyclically symmetric majority operation on the five-element set that yields a new kind of minimal clone.

The base set for our functions will be $\{0,1,2, \overline{1}, \overline{2}\}$; this notation will help to emphasize certain patterns in the functions. Table 3 shows the 26 majority operations in the clone under consideration. The functions are $f_{1}, f_{2}, g_{1}^{u, v}, \ldots, g_{6}^{u, v}$, 
where $u$ and $v$ can take the values $1, \overline{1}$ independently of each other. Thus each column $g_{i}^{u, v}$ represents 4 functions, giving altogether $2+6 \cdot 4=26$ functions. The first two functions are cyclically symmetric; we will use $f_{1}$ as a generator. We only list the tuples where the majority rule does not apply, and in the first five rows we make the same simplification as in Table 2 . For example, the row $\{0,1, \overline{1}\}$ indicates that any one of the 26 functions takes on the value 1 on any triple $(a, b, c)$ such that $\{a, b, c\}=\{0,1, \overline{1}\}$. Note also that for each of the functions the values on $(0, \overline{1}, 2),(0,1, \overline{2})$ and $(0,1,2)$ coincide, and the same holds for permutations of these triples.

The proof of Theorem 1 consists of three lemmas. First we prove that any majority operation generated by $f_{1}$ is one of the 26 functions appearing in Table 3 , then we verify that these functions indeed belong to $\left[f_{1}\right]$, and finally we prove that this clone is minimal.

Lemma 3. The clone generated by $f_{1}$ contains at most 26 majority operations.

Proof. Let $h$ be any majority function in $\left[f_{1}\right]$. We will examine restrictions of $h$ to three- and four-element subsets in order to prove that $h$ coincides with one of the 26 functions shown in Table 3 .

The three-element set $\{0,1, \overline{1}\}$ is preserved by $f_{1}$, and the restriction of $f_{1}$ to this set is isomorphic to $m_{1}$. There is only one majority function in [ $\left.m_{1}\right]$, therefore $f_{1}$ and $h$ coincide on $\{0,1, \overline{1}\}$. The same argument shows that the restriction of $h$ to any of the three-element sets shown in the first five rows of the table is uniquely determined.

The four-element set $\{\overline{1}, 0,2,1\}$ is also preserved by $f_{1}$, and the restriction to this set yields a function isomorphic to $M_{3}$ (see Table 2). This implies that there are 8 possibilities for $h$ on this four-element set, and $\left.h\right|_{\{\overline{1}, 0,2,1\}}$ is uniquely determined by $\left.h\right|_{\{0,1,2\}}$. Similarly, there are 8 possibilities for $h$ on $\{\overline{2}, 0,1,2\}$, and $\left.h\right|_{\{\overline{2}, 0,1,2\}}$ is uniquely determined by $\left.h\right|_{\{0,1,2\}}$.

Let us also observe that $h$ preserves $\{\overline{2}, 1, \overline{1}\}$, and its restriction to this set is isomorphic to $\left.h\right|_{\{0,1,2\}}$, hence the latter determines $\left.h\right|_{\{\overline{2}, 1, \overline{1}\}}$.

We see that most values of $h$ are determined by $\left.h\right|_{\{0,1,2\}}$, and the information we gathered about $h$ so far suffices to justify all the entries in Table 3 except for the ones in boldface. Ignoring these entries, i.e., the values on $\{0, \overline{1}, \overline{2}\}$, we have eight candidates for $h$, and the restrictions to $\{0,1,2\}$ uniquely determine these (yet partial) functions. Now we try to establish some relationships between the values on $\{0, \overline{1}, \overline{2}\}$ and $\{0,1,2\}$. To this end, we consider the smallest binary invariant relation $\rfloor^{2} \vartheta$ of $f_{1}$ relating 0 to 0,1 to $\overline{1}$ and 2 to $\overline{2}$ :

$$
\vartheta=\left\{\left(\begin{array}{l}
0 \\
0
\end{array}\right),\left(\begin{array}{l}
1 \\
1
\end{array}\right),\left(\begin{array}{l}
1 \\
\overline{1}
\end{array}\right),\left(\begin{array}{l}
2 \\
2
\end{array}\right),\left(\begin{array}{l}
2 \\
\overline{2}
\end{array}\right)\right\} .
$$

Similarly, let $\varrho$ be the smallest invariant relation of $f_{1}$ that relates 2 to 0,1 to $\overline{1}$ and 0 to $\overline{2}$ :

$$
\varrho=\left\{\left(\begin{array}{l}
0 \\
\overline{2}
\end{array}\right),\left(\begin{array}{l}
1 \\
1
\end{array}\right),\left(\begin{array}{l}
1 \\
\overline{1}
\end{array}\right),\left(\begin{array}{l}
1 \\
2
\end{array}\right),\left(\begin{array}{l}
2 \\
0
\end{array}\right),\left(\begin{array}{l}
2 \\
1
\end{array}\right),\left(\begin{array}{l}
2 \\
2
\end{array}\right)\right\} .
$$

\footnotetext{
${ }^{2}$ In other words, we consider the subalgebra generated by $\{(0,0),(1, \overline{1}),(2, \overline{2})\}$ in the direct square of the algebra $\left(\{0,1, \overline{1}, 2, \overline{2}\} ; f_{1}\right)$.
} 
We show below how the first one of the rows containing the boldface entries can be filled out with the help of these relations; the other five rows can be treated similarly.

Since $h$ belongs to $\left[f_{1}\right]$, it must preserve $\vartheta$ and $\varrho$, thus $h(0,1,2) \vartheta h(0, \overline{1}, \overline{2})$ and $h(2,1,0) \varrho h(0, \overline{1}, \overline{2})$. We already know that $h(0,1,2), h(2,1,0) \in\{1,2\}$, so we have the following four cases:

$$
h(0, \overline{1}, \overline{2})=\left\{\begin{aligned}
2, & \text { if } h(0,1,2)=2, h(2,1,0)=2 ; \\
2, & \text { if } h(0,1,2)=2, h(2,1,0)=1 \\
1, & \text { if } h(0,1,2)=1, h(2,1,0)=2 ; \\
1 \text { or } \overline{1}, & \text { if } h(0,1,2)=1, h(2,1,0)=1 .
\end{aligned}\right.
$$

We see that the value of $h(0, \overline{1}, \overline{2})$ is uniquely determined except for two of the eight possibilities (denoted by $\mathbf{u}$ and $\mathbf{v}$ in the table), where the value can be either 1 or $\overline{1}$.

Lemma 4. The clone generated by $f_{1}$ contains at least 26 majority operations.

Proof. We claim that the 26 functions shown in Table 3 belong to the clone generated by $f_{1}$. The function $f_{2}$ can be obtained from $f_{1}$ by permuting variables, and, similarly, $g_{i}^{u, v}$ can be obtained from $g_{1}^{u, v}$ for $i=2, \ldots, 6$. Thus it suffices to show that $g_{1}^{u, v} \in\left[f_{1}\right]$ for all $u, v \in\{1, \overline{1}\}$. This can be done by presenting a suitable composition for each of these four functions:

$$
\begin{aligned}
& g_{1}^{1,1}\left(x_{1}, x_{2}, x_{3}\right)=f_{1}\left(x_{2}, f_{1}\left(x_{2}, x_{1}, x_{3}\right), f_{1}\left(x_{1}, x_{2}, x_{3}\right)\right), \\
& g_{1}^{1, \overline{1}}\left(x_{1}, x_{2}, x_{3}\right)=f_{1}\left(x_{1}, x_{2}, f_{1}\left(x_{2}, x_{1}, x_{3}\right)\right), \\
& g_{1}^{\overline{1}, 1}\left(x_{1}, x_{2}, x_{3}\right)=f_{1}\left(x_{3}, x_{2}, f_{1}\left(x_{2}, f_{1}\left(x_{2}, x_{1}, x_{3}\right), f_{1}\left(x_{1}, x_{2}, x_{3}\right)\right)\right), \\
& g_{1}^{\overline{1}, \overline{1}}\left(x_{1}, x_{2}, x_{3}\right)=f_{1}\left(x_{3}, x_{2}, f_{1}\left(x_{1}, x_{2}, f_{1}\left(x_{2}, x_{1}, x_{3}\right)\right)\right) .
\end{aligned}
$$

Lemma 5. The clone generated by $f_{1}$ is minimal.

Proof. We need to verify that (2) holds for $f_{1}$, i.e., we have to prove that each of the 26 majority functions in $\left[f_{1}\right]$ generates $f_{1}$. Up to permutation of variables we have only the five functions $f_{1}, g_{1}^{1,1}, g_{1}^{1, \overline{1}}, g_{1}^{\overline{1}, 1}, g_{1}^{\overline{1}, \overline{1}}$. For the first one our task is void, for the remaining four ones the same composition works: for all $u, v \in\{1, \overline{1}\}$ we have

$$
f_{1}\left(x_{1}, x_{2}, x_{3}\right)=g_{1}^{u, v}\left(g_{1}^{u, v}\left(x_{2}, x_{1}, x_{3}\right), g_{1}^{u, v}\left(x_{1}, x_{3}, x_{2}\right), g_{1}^{u, v}\left(x_{3}, x_{2}, x_{1}\right)\right) .
$$

\section{Proof of Theorem 2}

Let $f$ be a cyclically symmetric majority operation on a set $A$ that generates a minimal clone containing $n$ majority operations. We add a new element to the base set: $A^{*}:=A \dot{\cup}\{*\}$, and we construct a majority function $f^{*}$ on $A^{*}$ as follows. If $a_{1}, a_{2}, a_{3} \in A^{*}$ are not pairwise different, then we define $f^{*}\left(a_{1}, a_{2}, a_{3}\right)$ by the majority rule, otherwise let

$$
f^{*}\left(a_{1}, a_{2}, a_{3}\right)=\left\{\begin{aligned}
f\left(a_{1}, a_{2}, a_{3}\right), & \text { if }\left\{a_{1}, a_{2}, a_{3}\right\} \subseteq A ; \\
a_{1}, & \text { if } * \in\left\{a_{1}, a_{2}, a_{3}\right\} .
\end{aligned}\right.
$$

Let us observe that $\left.f^{*}\right|_{A}$ coincides with $f$, and $\left.f^{*}\right|_{B}$ is isomorphic to $m_{2}$ for any three-element set $B \subseteq A^{*}$ that is not a subset of $A$. We claim that $f^{*}$ generates a minimal clone with $3 n$ majority operations. Just as in the previous section, we 
divide the task into three lemmas. First we prove that $3 n$ is an upper bound for the number of majority operations in $\left[f^{*}\right]$, then we show that this bound is sharp, and, finally, we verify that the clone is minimal.

Lemma 6. The clone generated by $f^{*}$ contains at most $3 n$ majority operations.

Proof. Let $h^{*}$ be any majority function in $\left[f^{*}\right]$. Since $f^{*}$ preserves $A$ and all threeelement subsets $B$ with $* \in B$, the function $h^{*}$ must preserve these sets as well. Clearly, $h^{*}$ is uniquely determined by its restrictions to all previously mentioned sets. The restriction of $h^{*}$ to $A$ belongs to $[f]$, hence there are $n$ possibilities for $\left.h^{*}\right|_{A}$. If $B$ is a three-element set containing $*$, then $\left.h^{*}\right|_{B}$ is isomorphic to one of the three majority functions in $\left[m_{2}\right]$. Moreover, if $B_{1}$ and $B_{2}$ are two such subsets, then $\left.h^{*}\right|_{B_{1}}$ and $\left.h^{*}\right|_{B_{2}}$ are isomorphic, since the same holds for $f^{*}$. This means that we cannot choose $\left.h^{*}\right|_{B_{1}}$ and $\left.h^{*}\right|_{B_{2}}$ independently: if one of them is given, the other one is uniquely determined. Thus $h^{*}$ is determined by $\left.h^{*}\right|_{A}$ and $\left.h^{*}\right|_{B}$ for one three-element set $B$ with $* \in B$, hence there are (at most) $3 n$ possibilities for the function $h^{*}$.

For the rest of the paper it will be convenient to introduce some notation. Let us rename the function $m_{2}$ to $d_{1}$, and let us denote the other two majority operations in its clone by $d_{2}$ and $d_{3}$ (see Table 1). The motivation for the notation is that $d_{i}$ coincides with the $i$ th projection whenever its arguments are pairwise different:

$$
d_{i}\left(a_{1}, a_{2}, a_{3}\right)=a_{i} \text { for }\left\{a_{1}, a_{2}, a_{3}\right\}=\{1,2,3\} .
$$

Observe also that $d_{3}$ is the dual discriminator function on $\{1,2,3\}$.

We have seen in the proof of the above lemma, that for any majority operation $h^{*} \in\left[f^{*}\right]$, the restriction of $h^{*}$ to $A$ is a majority function $h \in[f]$, and the restriction of $h^{*}$ to any three-element subset $B$ that contains $*$ is isomorphic to $d_{i}$ for some $i \in\{1,2,3\}$ (where $i$ does not depend on $B$ ). Since $h^{*}$ is determined by $h$ and $d_{i}$, we will use the notation $h^{*}=h * d_{i}$. For example, we have $f^{*}=f * d_{1}$. Equivalently, $h * d_{i}$ is the majority operation on $A^{*}$ defined for pairwise different $a_{1}, a_{2}, a_{3} \in A^{*}$ by

$$
\left(h * d_{i}\right)\left(a_{1}, a_{2}, a_{3}\right)=\left\{\begin{aligned}
h\left(a_{1}, a_{2}, a_{3}\right), & \text { if }\left\{a_{1}, a_{2}, a_{3}\right\} \subseteq A ; \\
a_{i}, & \text { if } * \in\left\{a_{1}, a_{2}, a_{3}\right\} .
\end{aligned}\right.
$$

In the following claim, which is the key for proving that $\left[f^{*}\right]$ contains at least $3 n$ majority operations, we will consider terms involving a ternary operation symbol $d$ and the variables $x_{1}, x_{2}, x_{3}$. We say that a term $s$ is obtained from the term $t$ using cyclic shifts, iff $s$ arises from $t$ by a finite number of replacements of some subterm $d\left(t_{1}, t_{2}, t_{3}\right)$ of $t$ by $d\left(t_{2}, t_{3}, t_{1}\right)$ or $d\left(t_{3}, t_{1}, t_{2}\right)$. More formally, the set CS $(t)$ of all terms that can be obtained from $t$ using cyclic shifts is defined inductively: If $t$ is a variable, then $\operatorname{CS}(t):=\{t\}$. Otherwise, if $t=d\left(t_{1}, t_{2}, t_{3}\right)$ where the sets $\operatorname{CS}\left(t_{i}\right)$, $i=1,2,3$, are already defined, then

$$
\mathrm{CS}(t):=\left\{d\left(s_{1}, s_{2}, s_{3}\right), d\left(s_{2}, s_{3}, s_{1}\right), d\left(s_{3}, s_{1}, s_{2}\right) \mid s_{i} \in \operatorname{CS}\left(t_{i}\right) \text { for } i=1,2,3\right\} .
$$

A straightforward induction argument shows that if one evaluates such terms over an algebra with a cyclically symmetric basic operation, then every term has the same term function as all its cyclically shifted descendants.

We are going to evaluate our terms over the algebra $\mathbb{B}=\left(\{1,2,3\} ; d_{1}\right)$. The term function corresponding to the term $t$ is denoted by $t^{\mathbb{B}}$. With this notation we have $d_{1}=\left(d\left(x_{1}, x_{2}, x_{3}\right)\right)^{\mathbb{B}}$, and $x_{i}^{\mathbb{B}}$ is the $i$ th ternary projection on $\{1,2,3\}$. (Let us note that we did not distinguish variables and projections until now.) 
Claim 7. If $t$ is a ternary term over the algebra $\mathbb{B}=\left(\{1,2,3\} ; d_{1}\right)$ and $t^{\mathbb{B}}$ is not a projection, then there exist terms $s_{1}, s_{2}, s_{3} \in \mathrm{CS}(t)$ such that

$$
s_{1}^{\mathbb{B}}=d_{1}, s_{2}^{\mathbb{B}}=d_{2} \text { and } s_{3}^{\mathbb{B}}=d_{3} .
$$

Proof. We prove the claim by term induction. Since $t$ does not evaluate to a projection, the term function corresponding to $t$ is a majority function. It is not hard to show (e.g., by another term induction) that after performing arbitrary cyclic shifts, we still get a majority operation, i.e., one of the functions $d_{1}, d_{2}, d_{3}$. We need to show that we can actually get all three of these functions.

Since $t$ cannot be a single variable, the initial step of the induction is the case when it contains only one operation symbol $d$. Then $t$ is of the form $d\left(x_{i_{1}}, x_{i_{2}}, x_{i_{3}}\right)$. The indices $i_{1}, i_{2}, i_{3}$ must be pairwise different (otherwise $t$ would evaluate to a projection), thus $t^{\mathbb{B}}=d_{i_{1}}$. Using cyclic shifts we get $\left(d\left(x_{i_{2}}, x_{i_{3}}, x_{i_{1}}\right)\right)^{\mathbb{B}}=d_{i_{2}}$ and $\left(d\left(x_{i_{3}}, x_{i_{1}}, x_{i_{2}}\right)\right)^{\mathbb{B}}=d_{i_{3}}$. Since $\left\{i_{1}, i_{2}, i_{3}\right\}=\{1,2,3\}$, we have obtained all three of $d_{1}, d_{2}, d_{3}$ (in some order).

For the inductive step let us write $t$ in the form $t=d\left(t_{1}, t_{2}, t_{3}\right)$. For each $k=1,2,3$ we have two possibilities for the subterm $t_{k}$ : either $t_{k}^{\mathbb{B}}=x_{i_{k}}^{\mathbb{B}}$ or $t_{k}^{\mathbb{B}}=d_{i_{k}}$ for some $i_{k} \in\{1,2,3\}$. By induction, in the latter case we can obtain any one of $d_{1}, d_{2}, d_{3}$ by performing cyclic shifts on $t_{k}$.

Suppose that some of $i_{1}, i_{2}, i_{3}$ coincide, say $i_{1}=i_{2} \neq i_{3}$. If $t_{1}^{\mathbb{B}}=t_{2}^{\mathbb{B}}=x_{i_{1}}^{\mathbb{B}}$, then $t^{\mathbb{B}}=\left(d\left(x_{i_{1}}, x_{i_{1}}, t_{3}\right)\right)^{\mathbb{B}}=x_{i_{1}}^{\mathbb{B}}$ by the majority rule, contradicting our assumption that $t$ does not evaluate to a projection. Thus at least one of $t_{1}^{\mathbb{B}}, t_{2}^{\mathbb{B}}$ equals $d_{i_{1}}$, say $t_{1}^{\mathbb{B}}=d_{i_{1}}$. Let us choose $j_{1} \in\{1,2,3\}$ to be different from $i_{2}$ and $i_{3}$, and construct a term $t_{1}^{\prime}$ using cyclic shifts in $t_{1}$ (by induction), such that $t_{1}^{\prime \mathbb{B}}=d_{j_{1}}$. For compatibility, let us put $j_{2}=i_{2}, j_{3}=i_{3}$ and $t_{2}^{\prime}=t_{2}, t_{3}^{\prime}=t_{3}$. The key property of the new terms $t_{1}^{\prime}, t_{2}^{\prime}, t_{3}^{\prime}$ is the following:

$$
t_{k}^{\prime} \in \mathrm{CS}\left(t_{k}\right), t_{k}^{\prime \mathbb{B}}=x_{j_{k}}^{\mathbb{B}} \text { or } t_{k}^{\mathbb{B}}=d_{j_{k}} \text {, and }\left\{j_{1}, j_{2}, j_{3}\right\}=\{1,2,3\} .
$$

If $i_{1}=i_{2}=i_{3}$, then at most one of $t_{1}, t_{2}, t_{3}$ can evaluate to a projection, and applying cyclic shifts on the other two terms we can still achieve (3). If $i_{1}, i_{2}, i_{3}$ are pairwise different, then (3) is trivially achieved by letting $j_{k}=i_{k}$ and $t_{k}^{\prime}=t_{k}$ for $k=1,2,3$.

Now we are in a position to write up the desired terms $s_{1}, s_{2}, s_{3}$ (keeping in mind that $\left.\left\{j_{1}, j_{2}, j_{3}\right\}=\{1,2,3\}\right)$ :

$$
\begin{aligned}
& s_{j_{1}}:=d\left(t_{1}^{\prime}, t_{2}^{\prime}, t_{3}^{\prime}\right) ; \\
& s_{j_{2}}:=d\left(t_{2}^{\prime}, t_{3}^{\prime}, t_{1}^{\prime}\right) ; \\
& s_{j_{3}}:=d\left(t_{3}^{\prime}, t_{1}^{\prime}, t_{2}^{\prime}\right) .
\end{aligned}
$$

These terms can be obtained from $t$ by cyclic shifts, therefore, the corresponding term functions are majority functions. Thus it suffices to verify the equalities $s_{j_{1}}^{\mathbb{B}}=d_{j_{1}}, s_{j_{2}}^{\mathbb{B}}=d_{j_{2}}, s_{j_{3}}^{\mathbb{B}}=d_{j_{3}}$ for tuples $\left(a_{1}, a_{2}, a_{3}\right) \in\{1,2,3\}^{3}$ where $a_{1}, a_{2}, a_{3}$ are pairwise different. For such a tuple we have $t_{k}^{\prime \mathbb{B}}\left(a_{1}, a_{2}, a_{3}\right)=a_{j_{k}}$, regardless of whether $t_{k}^{\prime \mathbb{B}}=x_{j_{k}}^{\mathbb{B}}$ or $t_{k}^{\prime \mathbb{B}}=d_{j_{k}}$, hence

$$
\begin{aligned}
& s_{j_{1}}^{\mathbb{B}}\left(a_{1}, a_{2}, a_{3}\right)=d_{1}\left(a_{j_{1}}, a_{j_{2}}, a_{j_{3}}\right)=a_{j_{1}}=d_{j_{1}}\left(a_{1}, a_{2}, a_{3}\right) \\
& s_{j_{2}}^{\mathbb{B}}\left(a_{1}, a_{2}, a_{3}\right)=d_{1}\left(a_{j_{2}}, a_{j_{3}}, a_{j_{1}}\right)=a_{j_{2}}=d_{j_{2}}\left(a_{1}, a_{2}, a_{3}\right) \\
& s_{j_{3}}^{\mathbb{B}}\left(a_{1}, a_{2}, a_{3}\right)=d_{1}\left(a_{j_{3}}, a_{j_{1}}, a_{j_{2}}\right)=a_{j_{3}}=d_{j_{3}}\left(a_{1}, a_{2}, a_{3}\right) .
\end{aligned}
$$


Let us observe that we did not really use the fact that $\mathbb{B}$ has only three elements: the claim is true for $\mathbb{B}=\left(B ; d_{1}\right)$ for an arbitrary nonempty set $B$, where $d_{1}$ is the majority operation defined for pairwise distinct $a_{1}, a_{2}, a_{3} \in B$ by the formula $d_{1}\left(a_{1}, a_{2}, a_{3}\right)=a_{1}$.

Lemma 8. The clone generated by $f^{*}$ contains at least $3 n$ majority operations.

Proof. We will prove that for any majority function $h \in[f]$, the three functions $h * d_{1}, h * d_{2}, h * d_{3}$ belong to $\left[f^{*}\right]$. Since $h \in[f]$, there is a composition of $f$ that yields $h$. This composition can be described by a ternary term $t$ such that the corresponding term function over the algebra $(A ; f)$ is $h$. Since $h$ is a majority operation, the term operation $t^{\mathbb{B}}$ over the algebra $\mathbb{B}:=\left(\{1,2,3\} ; d_{1}\right)$ is not a projection, and Claim 7 is applicable.

Let $s_{1}, s_{2}, s_{3}$ be the terms constructed from $t$ by cyclic shifts in Claim 7 , and let $h_{1}^{*}, h_{2}^{*}, h_{3}^{*}$ be the corresponding term functions over the algebra $\left(A^{*} ; f^{*}\right)$; these functions clearly belong to $\left[f^{*}\right]$. If $B$ is a three-element subset of $A^{*}$ that is not contained in $A$, then $\left.h_{i}^{*}\right|_{B}$ is isomorphic to $d_{i}$ as $\left(B ;\left.f^{*}\right|_{B}\right) \cong \mathbb{B}$. Since $\left.f^{*}\right|_{A}=f$ is cyclically symmetric, the cyclic shifts do not change the term functions on $A$ : we have $\left.h_{i}^{*}\right|_{A}=h$. Thus we can conclude that $h_{i}^{*}=h * d_{i}$ for $i=1,2,3$.

Since there are $n$ choices for $h$, the clone generated by $f^{*}$ contains the $3 n$ functions $h * d_{i}(h \in[f], i \in\{1,2,3\})$.

Remark 9. The previous two lemmas can be interpreted from the viewpoint of abstract clones as follows. For a fixed subset $B \subseteq A^{*}$ with $* \in B$, the restriction mappings

$$
\begin{aligned}
& \left.\right|_{A}:\left[f^{*}\right]^{(3)} \rightarrow\left[\left.f^{*}\right|_{A}\right]^{(3)}=[f]^{(3)} \\
& \left.\right|_{B}:\left[f^{*}\right]^{(3)} \rightarrow\left[\left.f^{*}\right|_{B}\right]^{(3)} \cong\left[m_{2}\right]^{(3)}
\end{aligned}
$$

are homomorphisms of Menger algebras (since they are induced by clone homomorphisms). The proof of Lemma 6 shows that the intersection of the kernels of these two homomorphisms is the equality relation on $\left[f^{*}\right]^{(3)}$, and the proof of Lemma 8 shows that these homomorphisms are surjective. Thus the Menger algebra $\left[f^{*}\right]^{(3)}$, which is decisive for the minimality of $\left[f^{*}\right]$, is a subdirect product of the Menger algebras $[f]^{(3)}$ and $\left[m_{2}\right]^{(3)}$.

Lemma 10. The clone generated by $f^{*}$ is minimal.

Proof. According to (2), we need to prove that for any majority operation $h^{*}$ in the clone generated by $f^{*}$, we have $f^{*} \in\left[h^{*}\right]$. We know that $h^{*}$ is of the form $h * d_{i}$, where $h \in[f]$ and $i \in\{1,2,3\}$. Since the clone generated by $f$ is minimal, there is a composition that produces $f$ from $h$. Applying this composition for $h^{*}=h * d_{i}$, we get a function of the form $f * d_{j}$. Taking into account that $f$ is cyclically symmetric, a suitable cyclic permutation of variables of $f * d_{j}$ yields $f^{*}=f * d_{1}$.

\section{ACKNOWLEDGEMENTS}

The authors would like to thank everyone that directly or indirectly contributed to the obtainment of the results presented in this article. In fact, these would not exist without many people that assisted in the computer search for cyclically symmetric minimal majority functions on a five-element set, and only few of which can be given credit here in a personal form.

In particular, the authors would like to mention Géza Makay for his recommendations concerning the design of the computer program that first found the minimal 
clone in Table 3 . Furthermore, they express their sincere gratitude towards the staff of the Department of Mathematics at the Technische Universität Dresden in general for the generous provision of computing capacities, and to Mr. Pierre Frison, who helped by running the program on his private laptop.

The second named author acknowledges that the present project is supported by the National Research Fund, Luxembourg, and cofunded under the Marie Curie Actions of the European Commission (FP7-COFUND), and supported by the Hungarian National Foundation for Scientific Research under grants no. K60148 and K77409.

\section{REFERENCES}

[1] B. Csákány, All minimal clones on the three-element set, Acta Cybernet. 6 (1983), no. 3, 227-238.

[2] B. Csákány, On conservative minimal operations, Lectures in Universal Algebra (Szeged, 1983), Colloq. Math. Soc. János Bolyai, 43, North-Holland, Amsterdam, 1986, 49-60.

[3] B. Csákány, Minimal clones - a minicourse, Algebra Universalis 54 (2005), no. 1, 73-89.

[4] B. Csákány, T. Gavalcová, Finite homogeneous algebras I, Acta Sci. Math. (Szeged) 42 (1980), no. 1-2, 57-65.

[5] E. Fried, A. F. Pixley, The dual discriminator function in universal algebra, Acta Sci. Math. (Szeged) 41 (1979), no. 1-2, 83-100.

[6] D. Lau, Function algebras on finite sets, Springer Monographs in Mathematics, SpringerVerlag, Berlin, 2006.

[7] K. Menger, The algebra of functions: past, present, future, Rend. Mat. Appl. V. Ser. 20 (1961), 409-430.

[8] R. Pöschel, L. A. Kalužnin, Funktionen- und Relationenalgebren, Mathematische Monographien, VEB Deutscher Verlag der Wissenschaften, Berlin, 1979. (German)

[9] R. W. Quackenbush, A survey of minimal clones, Aequationes Mathematicae 50 (1995), $3-16$.

[10] Á. Szendrei, Clones in Universal Algebra, Séminaire de Mathématiques Supérieures, 99, Presses de l'Université de Montréal, 1986.

[11] T. Waldhauser, Minimal clones generated by majority operations, Algebra Universalis 44 (2000), no. 1-2, 15-26.

[12] T. Waldhauser, Minimal clones with few majority operations, Acta Sci. Math. (Szeged) 73 (2007), no. 3-4, 471-486.

(M. Behrisch) Institut für Algebra, TU Dresden, D-01062 Dresden, Germany

E-mail address: mike.behrisch@mailbox.tu-dresden.de

(T. Waldhauser) University of Luxembourg, Mathematics Research Unit, 6 Rue Richard Coudenhove-Kalergi, L-1359 Luxembourg, Luxembourg, and, Bolyai Institute, UniverSity of Szeged, Aradi vértanúk tere 1, H-6720 Szeged, Hungary

E-mail address: twaldha@math.u-szeged.hu 